\title{
Results of a Double-Blind, Randomized Trial of Ceftobiprole Treatment of Complicated Skin and Skin Structure Infections Caused by Gram-Positive Bacteria ${ }^{\nabla}$
}

\author{
Gary J. Noel, ${ }^{1 *}$ Richard S. Strauss, ${ }^{1}$ Karen Amsler, ${ }^{1}$ Markus Heep, ${ }^{2}$ \\ Rienk Pypstra, ${ }^{2}$ and Joseph S. Solomkin ${ }^{3}$ \\ Johnson \& Johnson Pharmaceutical Research \& Development, L.L.C., Raritan, New Jersey ${ }^{1}$; Basilea Pharmaceutica Ltd., Basel, \\ Switzerland ${ }^{2}$; and University of Cincinnati College of Medicine, Cincinnati, Ohio ${ }^{3}$
}

Received 25 April 2007/Returned for modification 27 July 2007/Accepted 12 September 2007

\begin{abstract}
Ceftobiprole is the first broad-spectrum cephalosporin with activity against methicillin-resistant Staphylococcus aureus (MRSA) to be assessed in late-stage clinical trials. As a pivotal step in the clinical development of ceftobiprole, a multicenter, global, randomized, double-blind trial was conducted to compare the efficacy of ceftobiprole to that of vancomycin in patients with complicated skin and skin structure infections (cSSSIs) caused by gram-positive bacteria. The primary objective was to assess noninferiority on the basis of the cure rates 7 to 14 days after the completion of therapy in patients administered ceftobiprole $500 \mathrm{mg}$ every $12 \mathrm{~h}$ or vancomycin $1 \mathrm{~g}$ every $12 \mathrm{~h}$. Of 784 patients randomized, 282 receiving ceftobiprole and 277 receiving vancomycin were clinically evaluable. Of these patients, $93.3 \%$ treated with ceftobiprole and $93.5 \%$ treated with vancomycin were cured $(95 \%$ confidence interval of difference, $-4.4 \%, 3.9 \%)$. The cure rates for patients with MRSA infections were $91.8 \%(56 / 61)$ with ceftobiprole treatment and $90.0 \%(54 / 60)$ with vancomycin treatment (95\% confidence interval of difference, $-\mathbf{8 . 4 \%}, \mathbf{1 2 . 1 \%}$ ). At least one adverse event (AE) was reported by $52 \%$ of the ceftobiprole-treated patients and $51 \%$ of the vancomycin-treated patients. The most common AEs reported by the ceftobiprole-treated patients were nausea $(14 \%)$ and taste disturbance $(8 \%)$. Discontinuation of the study drug because of treatment-emergent AEs occurred in $4 \%(n=17)$ of the ceftobiprole-treated patients and $6 \%(n=22)$ of the vancomycin-treated patients. The results of this trial support the use of ceftobiprole as an effective and well-tolerated treatment option for patients with cSSSIs caused by a spectrum of gram-positive bacteria.
\end{abstract}

Complicated skin and skin structure infections (cSSSIs) in hospitalized patients are often caused by gram-positive bacteria (28). Because Staphylococcus aureus and Streptococcus pyogenes are the leading causes of cSSSIs, guidelines for the treatment of cSSSIs consistently recommend empirical antimicrobial therapy that provides activity against these bacteria.

Over the last 20 years, the prevalence of methicillin-resistant $S$. aureus (MRSA) as a cause of cSSSIs has been increasing worldwide $(15,18,19)$. Recent experience suggests that MRSA is now responsible for nearly $60 \%$ of purulent skin and soft tissue infections in patients presenting to emergency rooms in the United States (23). Community-origin MRSA with apparently enhanced virulence has emerged as the leading cause of serious skin infections arising in the community (23). The increasing importance of MRSA as a cause of cSSSIs has driven the need for the development of new agents with reliable activity against this pathogen. In particular, the development of a cephalosporin with a broad spectrum of activity that includes MRSA and that can be used to treat a range of serious polymicrobial infections began several years ago and has progressed to the point where one such agent, ceftobiprole, is now in late-stage clinical trials.

\footnotetext{
* Corresponding author. Mailing address: Johnson \& Johnson Pharmaceutical Research \& Development, L.L.C., 900 Route 202, Raritan, NJ 08869. Phone: (908) 704-4316. Fax: (908) 595-0843. E-mail: GNoel1 @ prdus.jnj.com.

Published ahead of print on 22 October 2007.
}

Ceftobiprole, an investigational novel pyrrolidinone cephalosporin, has the potential to be used as monotherapy for the treatment of patients with cSSSIs because of its activity against the major gram-negative and gram-positive bacteria, including MRSA, that cause these infections. Recent in vitro studies have indicated that the $\mathrm{MIC}_{90}$ for ceftobiprole is $\leq 2.0 \mu \mathrm{g} / \mathrm{ml}$ for MRSA $(11,16,17)$. In vivo studies with a number of animal models of infection have confirmed the potent in vitro activity of ceftobiprole $(5,7,12,17)$. After the completion of a phase II trial focused on assessing the efficacy of ceftobiprole for the treatment of patients with cSSSIs due to gram-positive bacteria (M. Heep, M., S. Querner, M. Harsch, and W. O'Riordan, Abstr. 44th Intersci. Conf. Antimicrob. Agents Chemother., poster L-361, 2004), a large, double-blind, randomized trial was conducted to compare the safety and efficacy of ceftobiprole to those of vancomycin, the current standard of care for the treatment of skin infections due to gram-positive bacteria in which methicillin resistance is a significant concern. We report here the results of that trial.

\section{MATERIALS AND METHODS}

This was a randomized, double-blind, multicenter trial that compared the efficacy and safety of ceftobiprole to those of vancomycin in patients with cSSSIs due to documented or suspected gram-positive pathogens.

Study population. Patients were eligible for the study if they were $\geq 18$ years old and were diagnosed with a cSSSI caused by documented or suspected grampositive bacteria. A cSSSI was defined as an infection involving subcutaneous tissues or requiring significant surgical intervention and one or more of the 
following: a wound infection, an abscess, or cellulitis. A wound infection was considered an infection that occurred within 30 days of surgery/trauma (including partial thickness burns covering $<10 \%$ of the body surface) with (i) purulent drainage from the lesion or (ii) at least three of the following signs: $(a)$ temperature $>38^{\circ} \mathrm{C}$ rectal $\left(\right.$ or $>37.5^{\circ} \mathrm{C}$ oral or $>38.5^{\circ} \mathrm{C}$ tympanic in the absence of antipyretics), $(b)$ localized swelling, $(c)$ localized erythema extending $\geq 10 \mathrm{~mm}$ beyond the wound edge, $(d)$ localized pain, or $(e)$ tenderness to palpation. An abscess (without an open wound) was one considered one that occurred in the 7 days before enrollment with purulent drainage or a purulent aspirate and (i) evidence of loculated fluid that required intervention within $48 \mathrm{~h}$ of enrollment and (ii) erythema and/or induration of $\geq 20 \mathrm{~mm}$ in diameter or tenderness. Cellulitis was considered cellulitis that occurred in the 7 days before enrollment with advancing edema, erythema, or induration and one of the following: (i) temperature $>38^{\circ} \mathrm{C}$ rectal, $>37.5^{\circ} \mathrm{C}$ oral, or $>38.5^{\circ} \mathrm{C}$ tympanic in the absence of antipyretics or reported fever in the 3 days before enrollment; (ii) a white blood cell count $\geq 10 \times 10^{9} /$ liter or $\geq 10 \%$ bands; and (iii) lymphangitis and adenopathy.

In addition to this characterization of infection at enrollment, blood was sampled for culture, the extent of tissue involvement (subcutaneous, fascia, muscle) was assessed, and the serum C-reactive protein concentration was measured.

Patients were excluded if they had a history of allergic reaction or intolerability to either cephalosporins or vancomycin, severe renal dysfunction (creatinine clearance $<30 \mathrm{ml} / \mathrm{min}$ or oliguria $<20 \mathrm{ml} / \mathrm{h}$ unresponsive to fluid challenge), hepatic dysfunction evident by serum transaminase levels greater than three times the upper limits of normal, or a condition that might affect adherence to the protocol requirements. Pregnant or lactating women, subjects with neutropenia, and human immunodeficiency virus-infected subjects with CD4 counts $<0.2 \times 10^{9}$ /liter were excluded from enrollment in this trial. Because the focus of this trial was on the treatment of infections caused by gram-positive bacteria and because diabetic patients with foot infections often develop infections that involve gram-negative bacteria, patients categorized as having diabetic foot infections were excluded from this trial. Patients with osteomyelitis or infections associated with animal or human bites were not enrolled in this trial. Patients who had received systemic antimicrobial therapy for more than $24 \mathrm{~h}$ in the 7 days before enrollment were excluded from participation in the study, with the exception of cases in which the infection was caused by microbiologically confirmed pathogens that were resistant to the previous antimicrobial agents or in which the subject was clinically worsening, as determined by the treating physician, despite at least $72 \mathrm{~h}$ of treatment.

Treatment was assigned by central randomization by using an automated clinical trial system. Randomization was balanced by using randomly permuted blocks and was stratified by infection type (surgery/trauma infection site, abscess, or cellulitis). Patients were stratified upon enrollment by the type of cSSSI (wound infection, abscess, cellulitis), and the enrollment of patients enrolled with cellulitis was limited to $20 \%$. The study assessments and evaluation criteria were consistent with guidelines from the Food and Drug Administration (1a) and the European Medicines Evaluation Agency (6) for clinical trials aimed at establishing an antibacterial agent as safe and effective for the treatment of patients with cSSSIs.

The trial design required the continuation of vancomycin therapy in the comparator arm rather than a switch to a semisynthetic penicillin in the absence of the identification of MRSA in the baseline culture to avoid the need to break the treatment blind. Additionally, conversion to oral therapy was not allowed, with the expectation that more severely ill patients would be enrolled in the trial and would require hospitalization for the duration of their antibiotic therapy.

Treatment. After enrollment into one of the protocol-defined disease strata (abscess, wound infection, cellulitis), the patients were randomly assigned in a 1:1 ratio to intravenous treatment with either ceftobiprole $500 \mathrm{mg}$ every $12 \mathrm{~h}$ or vancomycin $1 \mathrm{~g}$ every $12 \mathrm{~h}$ for 7 to 14 days. All subjects enrolled with the diagnosis of abscess required incision and drainage of the abscess either before or within $48 \mathrm{~h}$ of enrollment to meet the inclusion criteria. At the investigator's discretion, empirical therapy with aztreonam or metronidazole was permitted for the first $48 \mathrm{~h}$ of treatment, pending the identification of the organisms requiring this additional spectrum of activity. If such pathogens were not identified, this empirical therapy was stopped. No other systemic concomitant antibacterial treatment was allowed. Plasma vancomycin concentrations were monitored and adjusted on the basis of the individual investigators' local practices.

The double-blind trial design was maintained by assignment of an independent, unblinded pharmacist at each site who was responsible for study drug (vancomycin and ceftobiprole) preparation and for adjustment of the study drug doses on the basis of the individual subject's plasma vancomycin level or renal function. This activity was further monitored by an unblinded site monitor who operated independently of the blinded site monitor and all blinded activities.

Patients were evaluated before the start of therapy (baseline/predosing) and during therapy at visits on day $4 \pm 1$, day $8 \pm 1$, and day $14 \pm 1$ (if treatment beyond 14 days was deemed necessary). An end-of-therapy (EOT) visit was performed within $24 \mathrm{~h}$ after the last administration of the study drug. A test-ofcure (TOC) visit was performed 7 to 14 days after the EOT visit. Patients considered clinically cured at the TOC visit were evaluated for relapse at a late follow-up (LFU) visit 28 to 35 days after the EOT visit.

Clinical evaluation. The clinical evaluations (at the baseline, during treatment, and after treatment) included a microbiological assessment of the site of infection, evaluation of the signs and symptoms of infection, and at the TOC and LFU visits, an evaluation of the clinical outcome. The clinical outcome at the TOC visit, 7 to 14 days following the EOT visit, was categorized as cure, failure, or not evaluable. Cure was defined as a resolution of all signs and symptoms of the infection or improvement to such an extent that no further antimicrobial therapy was necessary. Failure was defined as a need for further treatment with a nonstudy antibiotic and discontinuation of the study drug due to a treatment-related AE or due to a lack of efficacy of the study drug after at least 3 days of study therapy. Patients who were assessed as failing therapy at the EOT visit were considered failures at the TOC visit. Patients who deviated from the protocol-defined treatment or evaluation procedures were considered not evaluable.

Clinical relapse was assessed at the LFU visit, 28 to 35 days after the EOT visit, only for patients who were cured at the TOC visit. Patients evaluated at the LFU visit were placed into the cure, clinical relapse, or not evaluable category. Cure occurred if no further antimicrobial therapy was necessary for the treatment of the primary infection site. Clinical relapse occurred if the signs and symptoms of the skin infection at the primary site reappeared and required additional antimicrobial therapy. Not evaluable was assigned if there was an absence of a clinical assessment at the LFU visit or because an antibiotic was administered concomitantly for a reason other than for treatment of the infection at the primary infection site.

Microbiological evaluations. The microbiological assessments included pathogen identification and susceptibility testing of all specimens, in addition to genotypic characterization of staphylococci (by testing for Panton-Valentine leukocidin [PVL] and $m e c A$ genes). Patients were assessed for microbiological outcome at the TOC visit, and this outcome was determined by Gram stain and culture of any discharge or infected tissues. The microbiological outcome for the patients was categorized as eradication, presumed eradication, colonization, persistence, presumed persistence, superinfection, or not evaluable. Patients were considered to have an eradicated microbiological outcome if no pathogen was isolated from any culture (fluid or tissue) taken at the original site of infection and to have a presumed eradicated microbiological outcome if no material suitable for culture was obtained from the primary site of infection in the absence of clinical signs or symptoms of infection. Patients were considered not evaluable with regard to microbiological outcome if no pathogen was isolated at the time of entry into the trial, no clinical evaluation was done at the TOC visit, no data related to microbiological outcome were collected at the TOC visit, or protocol-defined procedures were not conducted. Only patients with a microbiological outcome of eradication or presumed eradication at the TOC assessment were evaluated for relapse at the LFU assessment.

Bacteria were considered pathogens if they were isolated from the patients by sterile biopsy or aspiration in the presence of signs of inflammation. Duplicate isolates of all potential pathogens were sent to a central laboratory for antimicrobial susceptibility testing on customized TREK panels, according to CLSI methods and pathogen identification by coagulase and API system (bioMerieux) testing $(8,10)$. All isolates identified as staphylococci were tested for PVL and $m e c A$ genes by multiplex analyses, using a method similar to that described by McClure et al. (22). Isolates of staphylococci were categorized as methicillin resistant if they were shown to be oxacillin resistant according to CLSI criteria or were $m e c A$ positive (9).

Safety and tolerability. A treatment-emergent adverse event (AE) was any adverse change that occurred after a patient was given a study drug. Events involving exacerbations or the worsening of preexisting illnesses were recorded as AEs. A serious AE event was any experience that was life-threatening, that required hospitalization or prolonged hospitalization, or that resulted in death. Laboratory assessments were conducted at a central laboratory (Covance CLS).

Analysis populations. Patients were classified into the following populations for analyses: the intent-to-treat (ITT) population included all randomized patients; the modified intent-to-treat (mITT) population included all patients in the ITT population from whom a pathogen was isolated upon entry into the trial; the clinically evaluable (CE) population included all patients in the ITT population, 
TABLE 1. Patients included in each analysis population ${ }^{a}$

\begin{tabular}{lcc}
\hline \multirow{2}{*}{$\begin{array}{c}\text { Analysis } \\
\text { population }\end{array}$} & \multicolumn{2}{c}{ No. (\%) of patients } \\
\cline { 2 - 3 } & Ceftobiprole group & Vancomycin group \\
\hline ITT & $397(100)$ & $387(100)$ \\
mITT & $312(79)$ & $301(78)$ \\
CE & $282(71)$ & $277(72)$ \\
ME & $226(57)$ & $217(56)$ \\
Safety & $389(98)$ & $382(99)$ \\
\hline
\end{tabular}

${ }^{a}$ A total of 784 patients were evaluated.

excluding those with a clinical outcome of not evaluable at the TOC visit; and the microbiologically evaluable (ME) population included all patients in the $\mathrm{CE}$ population from whom a pathogen was isolated at the baseline, excluding those with a microbiological outcome of not evaluable at the TOC visit. Because this trial was focused on assessing the efficacy of ceftobiprole compared to that of vancomycin as a treatment for cSSSIs due to gram-positive bacteria, all subjects from whom a gram-negative pathogen was isolated at the baseline were excluded from the CE population. Cure in the mITT was assessed as occurring for patients from whom gram-positive and/or gram-negative bacteria were isolated at the baseline, provided that they received only protocol-defined study antibiotics (ceftobiprole, vancomycin, aztreonam, and metronidazole). The safety analysis population included all patients in the ITT population who were administered at least one dose of study medication.

Statistical considerations. This trial was designed to test the noninferiority of ceftobiprole treatment compared to the effectiveness of vancomycin treatment. The primary end point was the clinical cure rate at the TOC visit. The nul hypothesis was that the clinical cure rate for ceftobiprole-treated patients would be more than $10 \%$ inferior to the clinical cure rate for vancomycin-treated patients, and the alternative hypothesis was that the clinical cure rate for ceftobiprole-treated patients would not be more than $10 \%$ inferior to the clinical cure rate for vancomycin-treated patients. Similar hypotheses were applied to the analysis of the microbiological outcome.

The final sample size calculation was based on the confidence interval (CI) approach for a normal approximation of binomial probability. If clinical cure rates of $80 \%$ for both the ceftobiprole and the vancomycin treatment groups were assumed, with the limit of noninferiority of ceftobiprole treatment compared with the effectiveness of vancomycin treatment being $10 \%$ and with a power of $90 \%$ and a one-sided type I error of 0.025 , the estimated sample size of the clinically evaluable patients would be 276 per randomized group. If a nonevaluability rate of $30 \%$ is assumed, approximately 790 patients would be required so that ceftobiprole treatment could be concluded to be noninferior to vancomycin treatment if the lower limit of the $95 \%$ CI for the difference in the clinical cure rates (ceftobiprole minus vancomycin) was $\geq-10 \%$.

Clinical and microbiological outcomes were analyzed by the use of two-sided 95\% CIs for the between-treatment difference (ceftobiprole minus vancomycin) in the clinical cure rates and microbiological eradication rates at the TOC visit. The microbiological eradication rate and its CIs were summarized by infection type and initial pathogen.

\section{RESULTS}

Patient disposition. From October 2004 to December 2005, 129 study sites on five different continents (Europe, $n=45$; Asia, $n=34$; Africa, $n=8$; South America, $n=8$; North America, $n=34$ ) were initiated to participate in the trial, and 784 patients were randomized and enrolled in the trial. Of the 129 sites initiated, $101(78 \%)$ enrolled subjects. Approximately $42 \%$ of the patients $(328 / 784)$ randomized were enrolled at sites in the United States. In non-U.S. regions, approximately $36 \%, 15 \%, 4 \%$, and $3 \%$ of the subjects were enrolled in sites in Europe, Asia, Africa, and South America, respectively. Of the 784 randomized patients, 397 were assigned to the ceftobiprole arm and 387 were assigned to the vancomycin arm (Table 1).

Of all patients enrolled (ITT population), $85 \%$ completed the study per protocol. The most common causes of discontin-
TABLE 2. Demographics and baseline characteristics of ITT population

\begin{tabular}{|c|c|c|}
\hline Characteristic $^{a}$ & $\begin{array}{c}\text { Ceftobiprole } \\
\text { group } \\
(n=397)\end{array}$ & $\begin{array}{c}\text { Vancomycin } \\
\text { group } \\
(n=387)\end{array}$ \\
\hline Mean (SD) age (yr) & $48.0(16.1)$ & $46.7(16.3)$ \\
\hline Age range (yr) & $18-91$ & $18-89$ \\
\hline $\begin{array}{l}\text { No. }(\%) \text { of ITT population age } \\
>65 \mathrm{yr}\end{array}$ & $67(17)$ & $56(14)$ \\
\hline$\%$ Male:\% female & $55: 45$ & $61: 39$ \\
\hline Race (\% white:\% not white) & $76: 24$ & $76: 24$ \\
\hline Mean baseline wt (kg [SD]) & $81.0(21.76)$ & $82.7(24.2)$ \\
\hline $\begin{array}{l}\text { No. }(\%) \text { of ITT population with } \\
\text { lower-extremity primary } \\
\text { site of infection }\end{array}$ & $153(38.5)$ & $149(38.5)$ \\
\hline $\begin{array}{l}\text { No. (\%) of ITT population with } \\
\text { diabetes }\end{array}$ & $66(16.6)$ & $65(16.8)$ \\
\hline $\begin{array}{c}\text { Infection type (no. [\%] with } \\
\text { infection type) }\end{array}$ & 396 & 385 \\
\hline Abscess & $195(49)$ & $183(48)$ \\
\hline Wound & $127(32)$ & $132(34)$ \\
\hline Surgical wounds & 43 & 51 \\
\hline Traumatic wounds & 72 & 68 \\
\hline $\begin{array}{l}\text { Wounds associated with } \\
\text { burns }\end{array}$ & 12 & 13 \\
\hline Cellulitis & $74(19)$ & $70(18)$ \\
\hline
\end{tabular}

${ }^{a}$ The data are for 784 patients. No differences $(P>0.1$ by the chi-square test $)$ between the treatment groups were evident by testing for differences between the means or for differences in the distribution of groups.

uation were AEs or concomitant illnesses (35 patients), noncooperation (28 patients), and administrative issues (21 patients). The CE population included 282 patients in the ceftobiprole group and 277 patients in the vancomycin group. Among the 225 subjects in the ITT population who did not qualify for being clinically evaluable, 13 (8 in the ceftobiprole arm) were not given a study medication, 44 (19 in the ceftobiprole arm) were given an effective concomitant antibiotic therapy, 66 (37 in the ceftobiprole arm) received $<5$ days of therapy, 70 (36 in the ceftobiprole arm) had a gram-negative pathogen isolated at the baseline, 54 (27 in the ceftobiprole arm) were identified as having self-administered study drug, 6 ( 1 in the ceftobiprole arm) had an unconfirmed clinical diagnosis of a cSSSI, 2 ( 1 in the ceftobiprole arm) did not have a clinical evaluation at the TOC visit as defined in the protocol, and 19 (11 in the ceftobiprole arm) had a missing TOC visit in the absence of being reported to have a worsened condition at the EOT visit.

Demographics and baseline characteristics. The demographics and the baseline characteristics of the ITT patients were comparable between the ceftobiprole and vancomycin groups (Table 2). In the CE population, the proportion of men in the ceftobiprole arm (55\%) was significantly lower than the proportion of men in the vancomycin arm $(61 \%)(P=0.025)$. In the ITT population, $48 \%$ (378/781) of patients had abscesses, 33\% (259/781) had wounds, and 18\% (144/781) had 
TABLE 3. Distribution of gram-positive bacteria most frequently isolated at the baseline from the primary infection site in ceftobiprole- and vancomycin-treated subjects in the mITT and ME populations

\begin{tabular}{|c|c|c|c|c|}
\hline \multirow{3}{*}{ Organism } & \multicolumn{4}{|c|}{ No. $(\%)$ of all gram-positive isolates } \\
\hline & \multicolumn{2}{|c|}{ Isolates identified in mITT population } & \multicolumn{2}{|c|}{ Isolates identified in ME population } \\
\hline & Ceftobiprole group & Vancomycin group & Ceftobiprole group & Vancomycin group \\
\hline Staphylococcus aureus & $249(80.3)$ & $228(75.5)$ & $191(80)$ & $175(78.8)$ \\
\hline MRSA & $90(19.4)$ & $87(28.8)$ & $61(25.5)$ & $60(27)$ \\
\hline MSSA & $155(50)$ & $133(44)$ & $126(52.7)$ & $112(50.4)$ \\
\hline Strains with unknown susceptibility & $4(1.3)$ & $8(2.6)$ & $4(1.7)$ & $3(1.4)$ \\
\hline S. epidermidis ${ }^{a}$ & $8(2.5)$ & $13(4.3)$ & $8(3.3)$ & $9(4.0)$ \\
\hline Streptococcus pyogenes & $16(5.2)$ & $21(6.9)$ & $11(4.6)$ & $17(7.7)$ \\
\hline Streptococcus agalactiae & $5(1.6)$ & $5(1.6)$ & $3(1.3)$ & $1(0.5)$ \\
\hline Group G streptococcus & $3(1.0)$ & $6(2.0)$ & $1(0.4)$ & $6(2.7)$ \\
\hline Enterococcus faecalis & $5(1.6)$ & $3(1.0)$ & $5(2.1)$ & $3(1.4)$ \\
\hline
\end{tabular}

${ }^{a}$ S. epidermidis was identified by coagulase and API Staph testing.

cellulitis. No differences in the duration of treatment were observed between the treatment groups. For both treatment arms in the CE population, the mean durations of therapy were 9.2 days and the median durations of therapy were 8.0 days. Among the 559 subjects in the CE population, $30(5.4 \%)$ received either aztreonam or metronidazole, and these subjects were distributed similarly in each treatment arm (ceftobiprole, $n=13$; vancomycin, $n=17$ ).

The distribution of gram-positive bacteria isolated in the baseline cultures of samples from the infection site from patients in the mITT and the ME populations is shown in Table 3. In both the mITT and the ME populations, approximately $80 \%$ of the gram-positive bacteria isolated were S. aureus. MRSA isolates accounted for approximately one-third of all the $S$. aureus organisms isolated in these populations and approximately $25 \%$ of all gram-positive isolates recovered in this trial. Of the $477 \mathrm{~S}$. aureus isolates identified from patients in the mITT population, ceftobiprole and vancomycin MICs were determined for 461 . The $\mathrm{MIC}_{90} \mathrm{~s}$ for the $170 \mathrm{MRSA}$ isolates in this collection were $2 \mu \mathrm{g} / \mathrm{ml}$ (range, 0.5 to $2.0 \mathrm{mg} / \mathrm{liter}$; $\mathrm{MIC}_{50}$ and mode MIC, $0.5 \mu \mathrm{g} / \mathrm{ml}$ ) for ceftobiprole and $1 \mu \mathrm{g} / \mathrm{ml}$ (range, 0.5 to $2 \mu \mathrm{g} / \mathrm{ml} ; \mathrm{MIC}_{50}$ and mode MIC, $0.5 \mu \mathrm{g} / \mathrm{ml}$ ) for vancomycin. The $\mathrm{MIC}_{90} \mathrm{~s}$ for the 291 methicillin-susceptible Staphylococcus aureus (MSSA) isolates in this collection were $0.5 \mu \mathrm{g} / \mathrm{ml}$ (range, 0.12 to $1.0 \mu \mathrm{g} / \mathrm{ml} ; \mathrm{MIC}_{50}$ and mode $\mathrm{MIC}$, $0.25 \mu \mathrm{g} / \mathrm{ml}$ ) for ceftobiprole and $1 \mu \mathrm{g} / \mathrm{ml}$ (range, 0.5 to $2 \mu \mathrm{g} / \mathrm{ml}$; $\mathrm{MIC}_{50}$ and mode MIC, $0.5 \mu \mathrm{g} / \mathrm{ml}$ ) for vancomycin.

Seventy patients in the mITT population (34 randomized to the vancomycin treatment arm) had one or more gram-negative bacterial pathogens identified in their baseline culture, and only 8 (4 in each arm) of these patients were given aztreonam, in addition to the study drug regimen. The isolates identified in these patients included members of the family Enterobacteriaceae (Escherichia coli, Enterobacter spp., Klebsiella spp., Morganella morganii, Proteus mirabilis, Serratia marcescens), Acinetobacter spp., and Pseudomonas spp. In the 30 patients who had gram-negative bacteria identified in the baseline cultures and who received vancomycin alone, 14 (47\%) were assessed as cured at the TOC visit. This cure rate was considerably lower $(P<0.01)$ than the $81 \%$ cure rate $(26 / 32)$ observed for patients who received ceftobiprole alone in this analysis of the mITT population.

Outcomes. At the TOC visit, the clinical cure rates in the ceftobiprole-treated and vancomycin-treated patients were similar in both the ITT and the CE populations (Table 4). The $95 \%$ CI of the difference between the cure rates in the treatment arms was well within the $10 \%$ margin that was predefined as the criterion for the demonstration of noninferiority. The microbiological outcome of eradication or presumed eradication at the TOC visit in the microbiologically evaluable patients occurred in $94.2 \%$ of the ceftobiprole-treated patients and $93.5 \%$ of the vancomycin-treated patients $(95 \% \mathrm{CI},-3.8$, $5.2 \%$ ). Because of the potential for adjuvant surgical procedures to influence the cure of infection, the incidences of operative surgical intervention, topical wound care, and debridement procedures that occurred after the patient enrolled in the trial were also assessed across treatment groups. The

TABLE 4. Clinical cure rates in trial populations

\begin{tabular}{|c|c|c|c|}
\hline \multirow{2}{*}{ Population } & \multicolumn{2}{|c|}{$\begin{array}{l}\text { Cure rate (no. of } \\
\text { patients cured/total } \\
\text { no. of patients }[\%] \text { ) }\end{array}$} & \multirow{2}{*}{$\begin{array}{l}95 \% \text { CI of } \\
\text { difference } \\
\text { in cure } \\
\text { rates }\end{array}$} \\
\hline & $\begin{array}{l}\text { Ceftobiprole } \\
\text { group }\end{array}$ & $\begin{array}{l}\text { Vancomycin } \\
\text { group }\end{array}$ & \\
\hline ITT & $309 / 397(77.8)$ & $300 / 387(77.5)$ & $-5.5,6.1$ \\
\hline \multicolumn{4}{|l|}{ mITT } \\
\hline $\begin{array}{l}\text { Only gram-positive bacteria } \\
\text { isolated at the baseline }\end{array}$ & $225 / 276(81.5)$ & $219 / 267(82.0)$ & $-7.0,6.0$ \\
\hline $\begin{array}{l}\text { Only gram-negative bacteria } \\
\text { isolated at the baseline }\end{array}$ & $27 / 36(75)$ & $17 / 34^{a}(50)$ & $3.0,47.0$ \\
\hline CE with: & $263 / 282(93.3)$ & $259 / 277(93.5)$ & $-4.4,3.9$ \\
\hline Wound infections & $77 / 80(96.3)$ & $78 / 87(89.7)$ & $-1.0,14.2$ \\
\hline Abscess & $142 / 152(93.4)$ & $129 / 134(96.3)$ & $-7.9,2.2$ \\
\hline Cellulitis & $44 / 50(88.0)$ & $52 / 56(92.9)$ & $-16.2,6.4$ \\
\hline ME & $213 / 226(94.2)$ & $203 / 217(93.5)$ & $-3.8,5.2$ \\
\hline
\end{tabular}

${ }^{a}$ Cures occurred in 3 of 4 subjects who received aztreonam with vancomycin and 14 of 30 subjects who received vancomycin alone. 
TABLE 5. Clinical cure rates at TOC visit for patients in ME population with $S$. aureus infections

\begin{tabular}{lccc}
\hline & \multicolumn{2}{c}{$\begin{array}{c}\text { Cure rate (no. of } \\
\text { patients cured/total } \\
\text { no. of patients [\%]) }\end{array}$} & $\begin{array}{c}\text { 95\% CI of } \\
\text { Cause of infection }\end{array}$ \\
\cline { 2 - 3 } & $\begin{array}{c}\text { Ceftobiprole } \\
\text { group }\end{array}$ & $\begin{array}{c}\text { Vancomycin } \\
\text { group }\end{array}$ & \\
\hline All $S$. aureus isolates & $177 / 187(94.6)$ & $162 / 172(94.2)$ & $-0.4(-4.3,5.2)$ \\
MSSA & $121 / 126(96.0)$ & $108 / 112(96.4)$ & $-0.4(-5.2,4.4)$ \\
MRSA & $56 / 61(91.8)$ & $54 / 60(90.0)$ & $1.8(-8.4,12.1)$ \\
PVL positive + MRSA & $27 / 29(93.1)$ & $22 / 26(84.6)$ & \\
$\begin{array}{l}\text { MRSA and CRP }{ }^{a} \\
>50 \text { mg/dl }\end{array}$ & $20 / 23(86.9)$ & $21 / 24(87.5)$ & \\
$\begin{array}{l}\text { MRSA infections extending } \\
\text { to fascia or muscle }\end{array}$ & $19 / 21(90.5)$ & $18 / 21(85.7)$ & \\
\hline
\end{tabular}

${ }^{a} \mathrm{CRP}, \mathrm{C}$-reactive protein.

distribution of ceftobiprole-treated and vancomycin-treated patients with planned operative procedures $(24 \%$ and $28 \%$, respectively), unplanned surgical interventions $(6 \%$ and $5 \%$, respectively), topical dry dressing care $(60 \%$ and $63 \%$, respectively), topical wet dressing care ( $47 \%$ and $47 \%$, respectively), and debridement of tissues not in the operating room setting (15\% and $14 \%$, respectively) were similar.

Because of the importance of staphylococci in general and MRSA in particular as causes of cSSSIs, the outcomes for patients from whom $S$. aureus was isolated as a baseline pathogen were specifically assessed (Table 5). The overall clinical cure rates for patients with cSSSIs due to $S$. aureus were comparable for ceftobiprole-treated patients (94.6\% [177/187]) and vancomycin-treated patients $(94.2 \%$ [162/172]). The clinical cure rates were also comparable between ceftobiproletreated patients with MRSA infections (91.8\% [56/61]) and vancomycin-treated patients with MRSA infections $(90.0 \%$ [54/60]). The cure rates for patients who had deep infections due to MRSA were similar for the ceftobiprole-treated patients $(90.5 \%$ [19/21]) and the vancomycin-treated patients $(85.7 \%$ [18/21]).

The outcome of infections caused by PVL-positive isolates of $S$. aureus was analyzed because of observations that such isolates have been associated with more severe staphylococcal disease (23). The percentage of PVL-positive isolates varied considerably from region to region in this global trial and was the highest $(85.2 \%)$ among isolates from patients enrolled at sites in the United States. In the CE population, 40\% (144/359; 74 in the ceftobiprole treatment arm) of patients with $S$. aureus infections had PVL-positive isolates. Forty-five percent (55/ 121; 29 in the ceftobiprole treatment arm) of patients with MRSA infections had PVL-positive isolates. Although the differences in cure rates were not statistically significant, the cure rates were numerically higher among the ceftobiproletreated patients with MRSA isolates that were positive for PVL (93.1\% [27/29]) than among vancomycin-treated patients $(84.6 \%[22 / 26])$.

All patients with cSSSIs associated with $S$. aureus bacteremia (three ceftobiprole-treated patients, including two patients with MRSA infections, and two vancomycin-treated patients) were clinical cures, and the bacteremia resolved without complications.

The relapse rates in patients assessed to be cured at the
TABLE 6. Incidence of treatment-emergent AEs occurring in at least $3 \%$ of patients in either treatment group

\begin{tabular}{lcc}
\hline \multirow{2}{*}{ AE or characteristic } & \multicolumn{2}{c}{ No. (\%) of patients } \\
\cline { 2 - 3 } & $\begin{array}{c}\text { Ceftobiprole group } \\
(n=389)\end{array}$ & $\begin{array}{c}\text { Vancomycin group } \\
(n=382)\end{array}$ \\
\hline Nausea & $55(14)$ & $29(8)$ \\
Vomiting & $27(7)$ & $15(4)$ \\
Dysgeusia & $30(8)$ & $2(1)$ \\
Diarrhea & $21(5)$ & $15(4)$ \\
Constipation & $14(4)$ & $11(3)$ \\
Headache & $27(7)$ & $24(6)$ \\
Dizziness & $14(4)$ & $8(2)$ \\
Rash & $9(2)$ & $11(3)$ \\
Pruritus & $12(3)$ & $22(6)$ \\
At least one AE & $203(52)$ & $193(51)$ \\
At least one serious AE & $24(6)$ & $23(6)$ \\
Discontinuation due to AE & $17(4)$ & $22(6)$ \\
\hline
\end{tabular}

TOC visit were low for both ceftobiprole-treated and vancomycin-treated patients. The differences in the incidences of clinical relapse (1.6\% with ceftobiprole treatment versus $0.8 \%$ with vancomycin treatment; $95 \% \mathrm{CI},-1.2 \%, 2.7 \%$ ) and microbiological relapse $(2.7 \%$ with ceftobiprole treatment versus $5.3 \%$ with vancomycin treatment; $95 \% \mathrm{CI},-6.3 \%, 1.2 \%$ ) between treatment groups were not statistically significant. The clinical relapse rates were similar in the ceftobiprole-treated and the vancomycin-treated arms (four and two patients, respectively). Microbiological relapse appeared to be more common in the vancomycin-treated group (11 patients versus 6 patients in the ceftobiprole-treated group). A pathogen that was resistant to ceftobiprole or vancomycin was not isolated from any of the patients identified as having either a clinical or a microbiological relapse.

Safety and tolerability. No significant differences in the overall incidences of treatment-emergent AEs and discontinuations of study drugs due to AEs were observed between the treatment groups (Table 6). At least one AE was reported by $52 \%$ of the ceftobiprole-treated patients and by $51 \%$ of the vancomycin-treated patients. A total of 39 patients discontinued study medication due to an AE (17 treated with ceftobiprole and 22 treated with vancomycin). Among the ceftobiprole-treated patients, nausea (five patients) and vomiting (three patients) were the most frequent causes of the discontinuation of therapy. Among the vancomycin-treated patients, pruritus (four patients) and rash (three patients) were the most frequent reasons for the discontinuation of therapy.

Gastrointestinal events were reported in $25 \%$ of patients treated with ceftobiprole and $17 \%$ treated with vancomycin. Nausea, taste disturbance (dysgeusia), and vomiting were the AEs that were the most frequently reported by the ceftobiprole-treated patients and occurred in $14 \%, 8 \%$, and $7 \%$ of the patients, respectively. The AEs that were the most frequently reported by vancomycin-treated patients were nausea $(8 \%)$, headache $(6 \%)$, and pruritus $(6 \%)$. The difference between reports of nausea in the treatment arms was statistically significant. The majority of AEs reported by patients treated with both drugs were of mild or moderate intensity.

A total of 47 patients reported at least one serious $\mathrm{AE}$ during the course of the study: $24(6 \%)$ in the ceftobiprole 
TABLE 7. Marked treatment-emergent abnormalities in laboratory values that occurred in three or more subjects in either treatment group

\begin{tabular}{|c|c|c|c|c|c|c|}
\hline \multirow{4}{*}{ Laboratory test $^{a}$} & \multicolumn{6}{|c|}{ No. of patients } \\
\hline & \multicolumn{3}{|c|}{ Ceftobiprole group } & \multicolumn{3}{|c|}{ Vancomycin group } \\
\hline & \multirow{2}{*}{ Total $^{b}$} & \multicolumn{2}{|c|}{ Abnormality } & \multirow{2}{*}{ Total } & \multicolumn{2}{|c|}{ Abnormality } \\
\hline & & High & Low & & High & Low \\
\hline \multicolumn{7}{|l|}{ Chemistry } \\
\hline Creatinine $(2 \times$ ULN $)$ & 348 & 1 & & 350 & 4 & \\
\hline $\begin{array}{l}\text { Glucose }(>16.7 \mathrm{mmol} / \text { liter } \\
\text { and } \geq 100 \% \text { increase } \\
\text { or }<3.05 \mathrm{mmol} / \text { liter } \\
\geq 33 \% \text { decrease })\end{array}$ & 328 & 3 & 2 & 328 & 1 & 6 \\
\hline $\begin{array}{l}\text { Uric acid (>595 } \mu \mathrm{mol} / \\
\text { liter) }\end{array}$ & 348 & 6 & & 350 & 9 & \\
\hline $\operatorname{ALT}(>3 \times$ ULN $)$ & 321 & 5 & & 326 & 4 & \\
\hline AST (>3× ULN) & 310 & 2 & & 319 & 3 & \\
\hline GGT $(>3 \times$ ULN $)$ & 342 & 18 & & 350 & 21 & \\
\hline \multicolumn{7}{|l|}{ Hematology } \\
\hline $\begin{array}{l}\text { Eosinophil count } \\
\quad\left(>0.57 \times 10^{9} \text { cells/liter }\right. \\
\text { and } \geq 100 \% \text { increase })\end{array}$ & 289 & 6 & & 285 & 2 & \\
\hline $\begin{array}{l}\text { Neutrophil count }(>7.23 \\
\times 10^{9} \text { cells/liter and } \\
\geq 100 \text { increase or }<1.96 \\
\times 10^{9} \text { cells/liter and } \\
\geq 33 \% \text { decrease })\end{array}$ & 295 & 1 & 10 & 297 & 4 & 10 \\
\hline $\begin{array}{l}\text { Platelet count }(>600 \times \\
10^{9} \text { cells/liter and } \geq 50 \% \\
\text { increase or }<130 \times \\
10^{9} \text { cells/liter and } \geq 50 \% \\
\text { decrease) }\end{array}$ & 284 & 2 & 2 & 289 & 4 & 0 \\
\hline $\begin{array}{l}\text { White blood cell count } \\
(>15.0 \text { or }<3.8 \times \\
\left.10^{9} \text { cells/liter }\right)\end{array}$ & 297 & 6 & 0 & 297 & 9 & 0 \\
\hline
\end{tabular}

${ }^{a}$ ULN, upper limit of normal; ALT, alanine aminotransferase; AST, aspartate aminotransferase; GGT, $\gamma$-glutamyltransferase.

${ }^{b}$ Total number of subjects in whom analyte was measured.

group and $23(6 \%)$ in the vancomycin group. Three deaths occurred in the vancomycin treatment group, and none of these were assessed by the physicians caring for these patients to be related to the study drug or the failure of the study treatment. No deaths occurred in the ceftobiprole treatment group.

No differences in changes in mean laboratory testing values between the baseline and the end of therapy or in the incidence of abnormal values during therapy were found for hematologic measurements (total white blood cell count, differential, hemoglobin, red blood cell count, platelet count), biochemical measurements (serum electrolytes, blood urea nitrogen, creatinine, glucose, serum transaminases, bilirubin, alkaline phosphatase, lactate dehydrogenase, albumin, serum protein, uric acid, calcium, triglycerides, cholesterol), or urinalysis (hematuria, proteinuria, urinary sediment) when the results for the ceftobiprole- and the vancomycin-treated subjects were compared. The incidences of markedly abnormal test results for individual hematology and chemistry analytes within a given treatment group were low and were comparable for the two treatment groups (Table 7).

\section{DISCUSSION}

The results of this trial indicate that ceftobiprole is effective and noninferior to vancomycin for the treatment of cSSSIs caused by gram-positive pathogens. The clinical cure rate for the ceftobiprole-treated patients was $93.3 \%$ among those who were assessed as clinically evaluable 7 to 14 days after the completion of therapy. This result is consistent with the potent activity reported in vitro $(16,17)$, the results obtained with animal models of infection $(3,14)$, and the results obtained in a phase II trial (27; Heep et al., 44th ICAAC) reported previously. The earlier studies demonstrated that ceftobiprole has consistent activity against clinically important gram-positive pathogens, including staphylococci, a leading cause of serious skin and soft tissue infections $(4,12,17)$.

The design of this first large clinical trial involving assessment of the efficacy of ceftobiprole for the treatment of infections due to gram-positive pathogens considered an evolving understanding of the pharmacokinetics and the pharmacodynamics of this cephalosporin. The ceftobiprole dosing regimen was chosen on the basis of the pharmacokinetics of ceftobiprole studied in phase I and II trials and was estimated to provide plasma concentrations of free drug above $2 \mu \mathrm{g} / \mathrm{ml}$ for more than $30 \%$ of the dosing interval in over $90 \%$ of the patients (24). This $30 \%$ target was based on the findings of in vivo studies that indicated that the targets associated with the efficacy of ceftobiprole for the treatment of streptococcal and staphylococcal infections may be somewhat lower than what has been observed with other cephalosporins due to a moderate postantibiotic effect of ceftobiprole with these pathogens (1). For MSSA and MRSA isolates, the target pathogens in this trial, the probabilities of achieving free drug concentrations for at least $50 \%$ of the dosing interval for MSSA and MRSA were estimated to be $96.9 \%$ and $92.9 \%$, respectively (20). These estimates gave us sufficient confidence to proceed with this study of the assessment of the efficacy of ceftobiprole in patients with cSSSIs due to gram-positive pathogens with a dosing regimen of $500 \mathrm{mg}$ infused over $1 \mathrm{~h}$ and given every $12 \mathrm{~h}$. The relationship between achieving free drug concentrations and the clinical cure of infections caused by nonstaphylococcal organisms is expected to be different from that described for infections caused by staphylococcci, and this relationship is likely to require a percentage of time above the MIC for gram-negative bacteria greater than that required for staphylococci $(3,26)$. It was clear in designing the trial that the definition of efficacy in this experience would not be readily extrapolated to infections caused by members of the family Enterobacteriaceae and Pseudomonas aeruginosa, which, as demonstrated in this trial, are less frequent causes of cSSSIs.

This trial was designed to focus on the treatment of infections caused by gram-positive bacteria. Greater than $80 \%$ of the microbiologically evaluable patients were infected with staphylococci, and $30 \%$ of these patients were infected with methicillin-resistant pathogens. The distribution of methicillinresistant and methicillin-susceptible staphylococci in this study is consistent with the emerged predominance of MRSA as a cause of serious community-acquired and hospital-acquired skin infections recently reported in other trials $(21,29,30)$. Comparison with the results of trial experiences collected 5 to 10 years ago, in which similar inclusion and exclusion criteria 
were used $(2,25)$, highlights the changes in the epidemiology of MRSA as a causative pathogen in cSSSIs over the past decade. The high incidence of PVL-positive staphylococci adds an additional dimension to the snapshot of the current epidemiology that this study provides.

The study demonstrates the efficacy of ceftobiprole as a therapy for staphylococcal infections. The cure rates among patients with these infections consistently exceeded $90 \%$. The overall cure rate for the ceftobiprole-treated patients with staphylococcal infections was $94.6 \%$, and the cure rates were 91.8\% and $93.1 \%$ for patients with MRSA and PVL-positive MRSA infections, respectively. Although no statistical differences were seen between treatment groups in these subgroup analyses, point estimates of cure tended to be higher for the ceftobiprole-treated patients than for the vancomycin-treated patients. Taken together, these findings support the notion that the potent antistaphylococcal activity of ceftobiprole seen in vitro and in animal models will translate into effectiveness in clinic practice.

Clinical trials of newer anti-MRSA agents vary with regard to the mix of infections treated. In this trial, all subjects diagnosed with an abscess had an incision and drainage, and nearly $50 \%$ (51.2\% in the CE population and $48.2 \%$ in ITT population) had this procedure done after enrollment in the trial. Although the difference was not statistically significant, a higher cure rate in vancomycin-treated patients with abscesses $(96.3 \%)$ than in vancomycin-treated patients with wounds $(89.7 \%)$ and cellulitis $(92.9 \%)$ was observed. This trend has been observed in other clinical trials (13) and suggests that there are differences in cure rates among subgroups of patients enrolled in trials of treatments for cSSSIs. The overall cure rates for the clinically evaluable patients in both the ceftobiprole and the vancomycin arms of this trial were $90 \%$, and these cure rates align with those expected for an experience that was focused on infections caused by gram-positive bacteria, that excluded patients with diabetic foot infections, and in which the majority of patients had abscesses. Consistent with the lack of vancomycin's activity against gram-negative bacteria and ceftobiprole's broad spectrum of activity, the analysis of those patients in the mITT population from whom gram-negative bacteria were isolated at the baseline demonstrated that the cure rate observed for ceftobiprole-treated patients was better than that observed for vancomycin-treated patients. This observation demonstrates the expected behavior of the study population in regard to the benefit of effective antimicrobial therapy and suggests an additional benefit of ceftobiprole in patients found to have polymicrobial infections.

Another important contribution of this trial is the insight into the safety profile of ceftobiprole in a large sample of approximately 350 patients that was gained. Ceftobiprole was well tolerated by the majority of patients, and the frequency and severity of reports of AEs among the ceftobiprole-treated patients were comparable to those among vancomycin-treated patients. Reports of nausea and taste disturbance were significantly greater for the ceftobiprole-treated patients than for the vancomycin-treated patients. The majority of these events were transient and of mild or moderate severity. Treatment was discontinued due to an AE in only 4\% (Heep et al., 44th ICAAC) of the ceftobiprole-treated patients, whereas it was discontinued in $6 \%(21)$ of the vancomycin-treated patients.
The results of the trial described here demonstrate that ceftobiprole is a highly effective agent for the treatment of patients with infections due to a spectrum of gram-positive bacterial pathogens, including resistant staphylococci.

\section{ACKNOWLEDGMENT}

This study was supported by Johnson \& Johnson Pharmaceutical Research \& Development, L.L.C.

\section{REFERENCES}

1. Andes, D. R., and W. A. Craig. 2000. Abstr. 40th Intersci. Conf. Antimicrob. Agents Chemother., abstr. 1079.

1a.Anonymous. 1998. Guidance for industry. Uncomplicated and complicated skin and skin structure infections: developing antimicrobial drugs for treatment. Food and Drug Administration, Washington, DC. http://www.fda.gov /cder/guidance/2566dft.pdf.

2. Arbeit, R. D., D. Maki, F. P. Tally, E. Campanaro, and B. I. Eisenstein. 2004 The safety and efficacy of daptomycin for the treatment of complicated skin and skin-structure infections. Clin. Infect. Dis. 38:1673-1681.

3. Azoulay-Dupuis, E., J. P. Bedos, J. Mohler, A. Schmitt-Hoffmann, M. Schleimer, and S. Shapiro. 2004. Efficacy of BAL5788, a prodrug of cephalosporin BAL9141, in a mouse model of acute pneumococcal pneumonia. Antimicrob. Agents Chemother. 48:1105-1111.

4. Bogdanovich, T., C. Clark, L. Ednie, G. Lin, K. Smith, S. Shapiro, and P. C. Appelbaum. 2006. Activities of ceftobiprole, a novel broad-spectrum cephalosporin, against Haemophilus influenzae and Moraxella catarrhalis. Antimicrob. Agents Chemother. 50:2050-2057.

5. Bogdanovich, T., L. M. Ednie, S. Shapiro, and P. C. Appelbaum. 2005 Antistaphylococcal activity of ceftobiprole, a new broad-spectrum cephalosporin. Antimicrob. Agents Chemother. 49:4210-4219.

6. Carbon, C., G. B. Calandra, C. Norden, J. D. Nelson, and J. T. Mader. 1993. General guidelines for the evaluation of new anti-infective drugs for the treatment of selected infections of the skin and skin structure, p. 168-174. In T. R. Beam Jr., D. N. Gilbert, and C. M. Kunin (ed.), European guidelines for the clinical evaluation of anti-infective drug products. European Society of Clinical Microbiology and Infectious Diseases, Basel, Switzerland.

7. Chambers, H. F. 2005. Evaluation of ceftobiprole in a rabbit model of aortic valve endocarditis due to methicillin-resistant and vancomycin-intermediate Staphylococcus aureus. Antimicrob. Agents Chemother. 49:884-888.

8. CLSI. 2005. Methods for dilution antimicrobial susceptibility tests for bacteria that grow aerobically. M7-A6. CLSI, Wayne, PA

9. CLSI. 2005. Performance standards for antimicrobial disk susceptibility tests, 15th ed. M2-A8. CLSI, Wayne PA.

10. CLSI. 2005. Performance standards for antimicrobial susceptibility testing, 15th informational supplement. M100-S15. CLSI, Wayne, PA.

11. Denis, O., A. Deplano, C. Nonhoff, M. Hallin, R. De Ryck, R. Vanhoof, R. De Mendonca, and M. J. Struelens. 2006. In vitro activities of ceftobiprole, tigecycline, daptomycin, and 19 other antimicrobials against methicillin-resistant Staphylococcus aureus strains from a national survey of Belgian hospitals. Antimicrob. Agents Chemother. 50:2680-2685.

12. Deshpande, L. M., and R. N. Jones. 2003. Bactericidal activity and synergy studies of BAL9141, a novel pyrrolidinone-3-ylidenemethyl cephem, tested against streptococci, enterococci and methicillin-resistant staphylococci. Clin. Microbiol. Infect. 9:1120-1124.

13. Ellis-Grosse, E. J., T. Babinchak, N. Dartois, G. Rose, and E. Loh. 2005. The efficacy and safety of tigecycline in the treatment of skin and skin-structure infections: results of 2 double-blind phase 3 comparison studies with vancomycin-aztreonam. Clin. Infect. Dis. 41(Suppl. 5):S341-S353.

14. Entenza, J. M., P. Hohl, I. Heinze-Krauss, M. P. Glauser, and P. Moreillon. 2002. BAL9141, a novel extended-spectrum cephalosporin active against methicillin-resistant Staphylococcus aureus in treatment of experimental endocarditis. Antimicrob. Agents Chemother. 46:171-177.

15. Halem, M., J. Trent, J. Green, and F. Kerdel. 2006. Community-acquired methicillin resistant Staphylococcus aureus skin infection. Semin. Cutan. Med. Surg. 25:68-71.

16. Hebeisen, P., I. Heinze-Krauss, P. Angehrn, P. Hohl, M. G. Page, and R. L. Then. 2001. In vitro and in vivo properties of Ro 63-9141, a novel broadspectrum cephalosporin with activity against methicillin-resistant staphylococci. Antimicrob. Agents Chemother. 45:825-836.

17. Jones, R. N., L. M. Deshpande, A. H. Mutnick, and D. J. Biedenbach. 2002. In vitro evaluation of BAL9141, a novel parenteral cephalosporin active against oxacillin-resistant staphylococci. J. Antimicrob. Chemother. 50:915932 .

18. Kluytmans-Vandenbergh, M. F., and J. A. Kluytmans. 2006. Communityacquired methicillin-resistant Staphylococcus aureus: current perspectives. Clin. Microbiol. Infect. 12(Suppl. 1):9-15.

19. Kollef, M. H., and S. T. Micek. 2006. Methicillin-resistant Staphylococcus aureus: a new community-acquired pathogen? Curr. Opin. Infect. Dis. 19: $161-168$. 
20. Lodise, T. P., Jr., R. Pypstra, J. B. Kahn, B. P. Murthy, H. C. Kimko, K. Bush, G. J. Noel, and G. L. Drusano. 2007. Probability of target attainment for ceftobiprole as derived from a population pharmacokinetic analysis of 150 subjects. Antimicrob. Agents Chemother. 51:2378-2387.

21. Martone, W. J., and K. C. Lamp. 2006. Efficacy of daptomycin in complicated skin and skin-structure infections due to methicillin-sensitive and -resistant Staphylococcus aureus: results from the CORE Registry. Curr. Med. Res. Opin. 22:2337-2343.

22. McClure, J. A., J. M. Conly, V. Lau, S. Elsayed, T. Louie, W. Hutchins, and K. Zhang. 2006. Novel multiplex PCR assay for detection of the staphylococcal virulence marker Panton-Valentine leukocidin genes and simultaneous discrimination of methicillin-susceptible from -resistant staphylococci. J. Clin. Microbiol. 44:1141-1144.

23. Moran, G. J., A. Krishnadasan, R. J. Gorwitz, G. E. Fosheim, L. K. McDougal, R. B. Carey, and D. A. Talan. 2006. Methicillin-resistant $S$. aureus infections among patients in the emergency department. N. Engl. J. Med. 355:666-674.

24. Mouton, J. W., A. Schmitt-Hoffmann, S. Shapiro, N. Nashed, and N. C. Punt. 2004. Use of Monte Carlo simulations to select therapeutic doses and provisional breakpoints of BAL9141. Antimicrob. Agents Chemother. 48:17131718.

25. Nichols, R. L., D. R. Graham, S. L. Barriere, A. Rodgers, S. E. Wilson, M Zervos, D. L. Dunn, B. Kreter, et al. 1999. Treatment of hospitalized patients with complicated gram-positive skin and skin structure infections: two ran- domized, multicentre studies of quinupristin/dalfopristin versus cefazolin, oxacillin or vancomycin. J. Antimicrob. Chemother. 44:263-273.

26. Sanchez-Navarro, A., C. I. Colino, and M. M. Sanchez Recio. 2001. A retrospective analysis of pharmacokinetic-pharmacodynamic parameters as indicators of the clinical efficacy of ceftizoxime. Clin. Pharmacokinet. 40: 125-134.

27. Schmitt-Hoffmann, A., M. Harsch, M. Heep, M. Schleimer, T. Brown, and A. Man. 2004. BAL5788 in patients with complicated skin and skin structure infections caused by gram-positive pathogens including methicillin-resistant Staphylococcus species (MRSS), abstr. P-1031. Abstr. 14th ECCMID.

28. Stevens, D. L., A. L. Bisno, H. F. Chambers, E. D. Everett, P. Dellinger, E. J. Goldstein, S. L. Gorbach, J. V. Hirschmann, E. L. Kaplan, J. G. Montoya, and J. C. Wade. 2005. Practice guidelines for the diagnosis and management of skin and soft-tissue infections. Clin. Infect. Dis. 41:1373-1406.

29. Stryjewski, M. E., V. H. Chu, W. D. O'Riordan, B. L. Warren, L. M. Dunbar, D. M. Young, M. Vallee, V. G. Fowler, Jr., J. Morganroth, S. L. Barriere, M. M. Kitt, and G. R. Corey. 2006. Telavancin versus standard therapy for treatment of complicated skin and skin structure infections caused by grampositive bacteria: FAST 2 study. Antimicrob. Agents Chemother. 50:862867.

30. Weigelt, J., K. Itani, D. Stevens, W. Lau, M. Dryden, and C. Knirsch. 2005. Linezolid versus vancomycin in treatment of complicated skin and soft tissue infections. Antimicrob. Agents Chemother. 49:2260-2266. 